\title{
Retrieval of phase history parameters from distributed scatterers in urban areas using very high resolution SAR data
}

\author{
Yuanyuan Wang a,*, Xiao Xiang Zhu ${ }^{\mathrm{a}, \mathrm{b}}$, Richard Bamler ${ }^{\mathrm{a}, \mathrm{b}}$ \\ a Lehrstuhl für Methodik der Fernerkundung, Technische Universität München, Arcisstraße 21, 80333 Munich, Germany \\ ${ }^{\mathrm{b}}$ Remote Sensing Technology Institute (IMF), German Aerospace Center (DLR), Oberpfaffenhofen, 82234 Wessling, Germany
}

\section{A R T I C L E I N F O}

Article history:

Available online 1 August 2012

\section{Keywords:}

Phase history

Distributed scatterer

Covariance matrix

SAR

PSI

TerraSAR-X

\begin{abstract}
A B S T R A C T
In a recent contribution Ferretti and co-workers (Ferretti, A., Fumagalli, A., Novali, F., Prati, C., Rocca, F., Rucci, A., 2011. A new algorithm for processing interferometric data-stacks: SqueeSAR IEEE Transactions on Geoscience and Remote Sensing 49(9), pp. 3460-3470) have proposed the SqueeSAR method, a way to exploit temporally coherent distributed scatterers in coherent SAR data stacks. Elevation and deformation or subsidence estimates are obtained with accuracy similar as in the well known persistent scatterer interferometry (PSI).

In this paper we propose an alternative approach and provide a first demonstration of the optimal estimation of distributed scatterers' phase histories in urban areas. Different to SqueeSAR, we derive phase histories for each distributed scatterer pixel rather than for groups of pixels. We use the AndersonDarling statistical test to identify neighboring samples of the same distribution. Prior to covariance matrix estimation required for maximum likelihood estimation we apply a multi-resolution defringe technique. By using TerraSAR-X high resolution spotlight data, it is demonstrated that we are able to retrieve reliable phase histories and motion parameter estimates from distributed scatterers with signal-to-noise-ratio far below the common range.

() 2012 International Society for Photogrammetry and Remote Sensing, Inc. (ISPRS) Published by Elsevier B.V. All rights reserved.
\end{abstract}

\section{Introduction}

Persistent scatterer interferometry (PSI) (Ferretti et al., 2001; Kampes, 2006) exploits the phase history of strong and long term stable scatterers, i.e. the so-called persistent scatterers (PSs), over the entire period of monitoring. Commonly, PSs are selected according to their amplitude dispersion index or their signal-toclutter ratio (SCR). They are usually seen as bright pixels in synthetic aperture radar (SAR) images. However these criteria are only indications but not necessarily measurements of the decorrelation. Common PSs in real world are stable objects such as window sills on building façades, building corners, and exposed rocks. By proper modeling the phase histories of PSs, the elevation and the time series of the deformation of each PS can be estimated. PSI can provide both large-scale and long-term deformation monitoring with an accuracy of up to $\mathrm{mm} / \mathrm{year}$. This is particularly true for urban areas where the PS density is high. As a price for its efficiency, first, PSI considers only a single dominant target within a SAR resolution cell for each PS which forbids layover separation; and second, only permanently reliable scatterers are exploited. This makes PSI an

\footnotetext{
* Corresponding author. Tel.: +49815328 2386.

E-mail address: yuanyuan.wang@dlr.de (Y. Wang).
}

opportunistic approach and functionally limits the density of the useful scatterers.

The following development of SAR interferometry (InSAR) techniques basically targets at understanding the interaction of the SAR signal within a resolution cell, and at optimally exploring coherent objects other than detected PSs, such as layovered scatterers and distributed scatterers (DSs).

In the first aspect, the authors of (Ferretti et al., 2001) extended PSI by considering phase models for multiple scatterers within a pixel (Ferretti et al., 2005). Or to be more general, differential SAR tomography (D-TomoSAR) (Lombardini, 2005; Fornaro et al., 2009; Zhu and Bamler, 2010, 2011) provides a general solution for such case and avoids the pre-selection of coherent scatterers. D-TomoSAR can separate multiple scatterers at different elevation with possible motion inside a pixel by means of spectral estimation. D-TomoSAR is able to retrieve topography, deformation parameters, or even the entire phase history of multiple scatterers within a resolution cell. Yet compared to PSI, D-TomoSAR is relatively computationally expensive. In the second aspect, the small baseline subset (SBAS) technique (Casu et al., 2005) overcomes the second disadvantage of PSI by further exploiting "temporary" PSs. That is to say, instead of looking for permanently coherent scatterers with respect to a single master acquisition, SBAS takes 
advantage of high coherence in pairs of acquisitions with small baselines (both temporal and spatial). In a popular notion, SBAS builds up interferograms between "good" pairs of acquisitions. Then the processing of each set of interferograms with a common master follows standard PSI procedures. Based on these differential results from numbers of interferograms, an inversion of an overdetermined linear equation system gives a complete time series of the phase history of a scatterer. SBAS theoretically increases the density of useful scatterers, and decreases the number of images required (Casu et al., 2005).

However, in non-urban environment where surface or volume scattering dominates, PSs density can be very low and DSs are the majority. Even in urban areas, asphalt roads and concrete surfaces seen as DS in X-band also make up a significant percentage. The question then comes to optimally harvest the information from a DS, i.e. how to retrieve its phase history, and model parameters such as elevation and deformation velocity. The challenges are the following. DSs tend to decorrelate temporally, i.e. interferometric DS pixels have usually much higher phase noise than PSs. Also decorrelation may be different for each of the interferograms in the stack.

The SqueeSAR technique (Ferretti et al., 2011a, 2011b) provides a possible solution for these problems. It uses the fact that a DS pixel is usually surrounded by many pixels from the same distribution, also called "brother pixels" in (Ferretti et al., 2011a). SqueeSAR detects these brotherhood segments, uses them for estimation of the covariance matrix and obtains the maximum likelihood estimation (MLE) of the phase history of the segment (De Zan and Rocca, 2005; Seymour and Cumming, 1994). Since SqueeSAR treats each brotherhood segment as a single object, spatial resolution of the estimated phase history is compromised.

This article presents the first demonstration of exploiting DS for urban monitoring using very high resolution SAR image stacks. We estimate elevation and motion history of each DS pixel in an optimum way. Following the basic concept of SqueeSAR, we introduce several new aspects:

- Instead of the brotherhood segments as single objects we retrieve the phase history for each DS pixel.

- For identifying the brotherhood pixels we use a more robust statistical test, i.e. the Anderson-Darlington (AD) test.

- For covariance matrix estimation we introduce a multi-resolution interferometric phase flattening (defringe) algorithm to determine the underlying topographic and motion-induced phase components.

The remainder of this article starts with the explanation of our covariance matrix estimation procedures, followed by phase history retrieval and its model parameters estimation by MLE. Results using TerraSAR-X (TS-X) high resolution spotlight data are present as last.

\section{Covariance matrix estimation}

The covariance between two single-look complex (SLC) measurements $i_{n}$ and $i_{k}$ in image $n$ and $k$ is defined as the expectation of the product of one with the complex conjugate of the other:

$c_{n, k}=E\left(i_{n} i_{k}^{*}\right)$

Normalizing the covariance by its standard deviation yields the complex correlation coefficients, usually referred to as coherence (Bamler and Hartl, 1998):

$\gamma_{n, k}=\frac{E\left(i_{n} i_{k}^{*}\right)}{E\left(\left|i_{n}\right|\right) E\left(\left|i_{k}\right|\right)}$
The MLE of the covariance is obtained by assuming ergodicity and replacing the expectation with averaging $M$ independent samples:

$\hat{c}_{n, k}=\frac{1}{\mathrm{M}} \sum_{m} i_{n}(m) i_{k}^{*}(m)$

and for coherence:

$\hat{\gamma}_{n, k}=\frac{\sum_{m} i_{n}(m) i_{k}^{*}(m)}{\sqrt{\sum_{m}\left|i_{n}(m)\right|^{2} \sum_{m}\left|i_{k}(m)\right|^{2}}}$

The covariance matrix $\mathbf{C}$ containing all the covariances is then

$\mathbf{C}=\mathbf{Z Z}^{\mathrm{H}}$

where

$\mathbf{Z}=\left[\begin{array}{cccc}i_{1}(1) & i_{1}(2) & \cdots & i_{1}(\mathrm{M}) \\ i_{2}(1) & i_{2}(2) & \cdots & i_{2}(\mathrm{M}) \\ \vdots & & \ddots & \vdots \\ i_{\mathrm{N}}(1) & i_{\mathrm{N}}(2) & \cdots & i_{\mathrm{N}}(\mathrm{M})\end{array}\right]$

However, this estimator is biased at low magnitudes of coherence for small $M$ (Touzi et al., 1999; Bamler and Hartl, 1998) According to the Reed-Mallett-Brennan (RMB) detection loss theory (Reed et al., 1974), to maintain a loss of likelihood (see Eq. (7)) of the estimates of less than $3 \mathrm{~dB}, \mathrm{M}$ should be larger than $2 N$, where $N$ is the number of measurements:

loss $=-10 \log _{10}[(M+2-N) /(M+1)]$

In our experiment, we try to ensure $M=3 N$ as much as possible. For low coherence bias correction, we adopt a method explained in (Zebker and Chen, 2005) which makes use of an empirical function of estimated coherence with respect to the theoretical one.

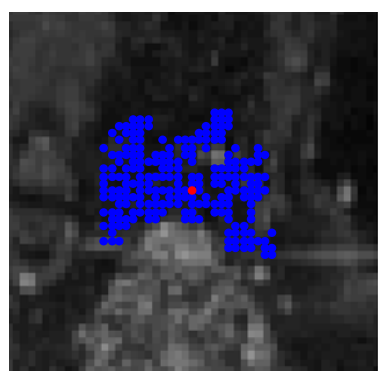

(a)
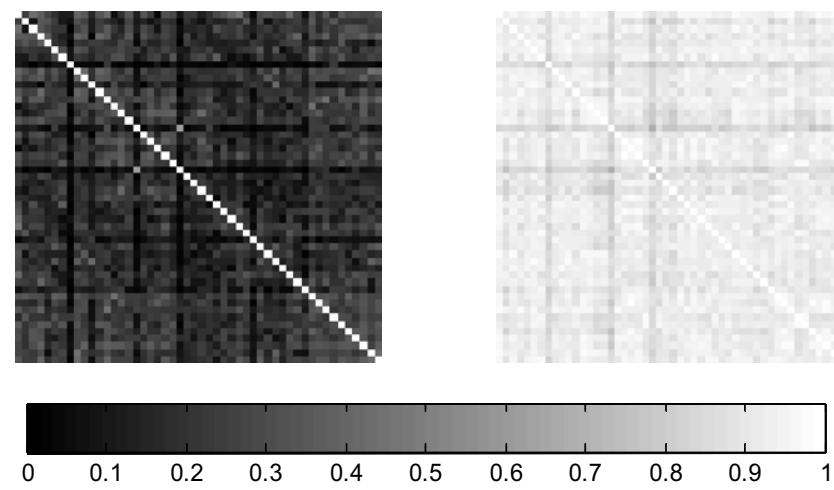

(c)

(d)
Fig. 1. (a) and (b): Samples selected by adaptive and rectangle sample selection strategy, respectively. The red dot is the target pixel, while those in blue are the selected pixels, i.e. The neighboring pixels sharing the same statistical distribution as the target pixel. (c) and (d): Corresponding coherence matrices. 


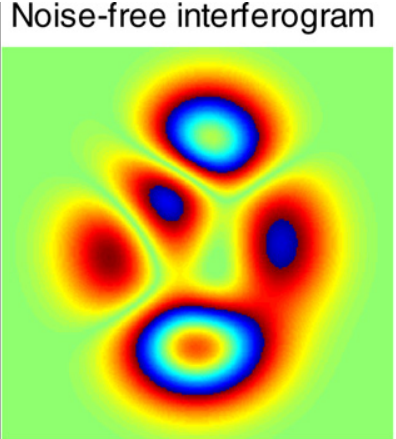

(a)

Multi-Resolution patch size

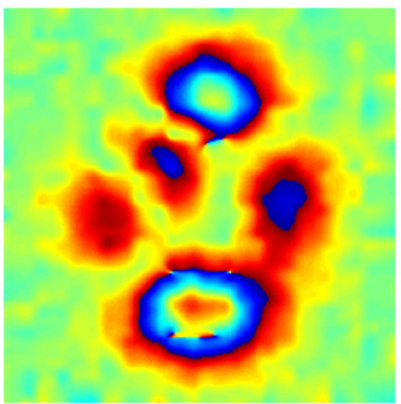

(c)

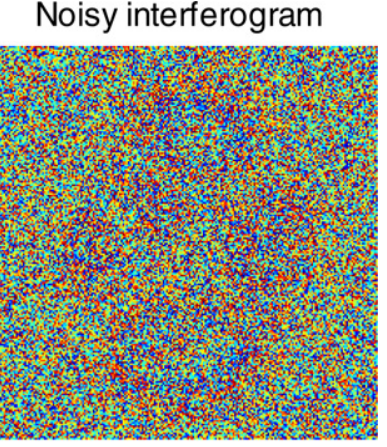

(b)

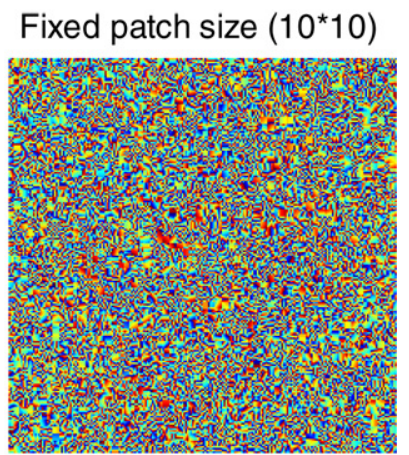

(d)
Fig. 2. (a) Simulated noise-free interferogram, (b) simulated noisy interferogram. $30 \%$ Of the pixels are DS with coherence $=0.3$ corresponding to a SNR of $-3.7 \mathrm{~dB}$ in the two SLC images, and the rest $70 \%$ are noisy pixels with coherence close to 0 , (c) fringes estimated by adaptive multi-resolution method, and (d) fringes estimated using single fixed patch size $(10 \times 10$ pixels $)$.

Other than this systematic drawback of the covariance estimator, it still faces two major practical issues. Especially in high resolution SAR images of urban areas, the ergodicity of the selected samples is hardly ensured. This jeopardizes the covariance estimation in two ways. First, carelessly selecting these samples, such as using a simple rectangular estimation window (commonly known as box-car method) brings the risk of averaging pixels of different distributions, and hence different objects, yielding a wrong estimate of the covariance matrix. And second, the spatially varying phase within the estimation window caused by topography or motion diminishes the summation in Eqs. (3) and (4), leading to an underestimation of the covariance or coherence. Therefore, this phase needs to be removed before the covariance estimation. The covariance estimator in Eqn. (3) then becomes:

$\hat{c}_{n, k}=\frac{1}{M} \sum_{m} i_{n}(m) i_{k}^{*}(m) \exp \left(-j\left(\bar{\phi}_{n}(m)+\bar{\phi}_{k}(m)\right)\right)$

where $\bar{\phi}_{n}$ and $\bar{\phi}_{k}$ are the expected phases of $i_{n}$ and $i_{k}$.

In Eq. (3), the phase of $c_{n, k}$ is the expected phase difference of $i_{n}$ and $i_{k}$. On one hand, we must remove the deterministic part of the phase of $i_{n}$ and $i_{k}$ for better covariance estimation, and on the other hand we are exploring this interferometric phase from $c_{n, k}$ for model parameters estimation.

To summarize, the covariance estimation procedure is a twostep approach: (1) adaptive sample selection by AD test on the amplitudes of the samples, and (2) multi-resolution defringe to remove the underlying expectation of the phases. These two steps will be discussed in detail in the following two sections.

\subsection{Adaptive sample selection}

The complex response of a DS in a SAR image is commonly modeled as circular Gaussian variate that widely holds for medium or lower resolution SAR data. However, due to limited number of natural scatterers in a resolution cell in high resolution SAR systems, not fully developed speckle results an imperfect circular Gaussian distribution. Yet we still favor the Gaussian model, as the best asymptotical one, for conveniences in the estimation (i.e. only the covariance is needed).

The MLE of each DS pixel value in the stack is found via inversion of its covariance matrix. As mentioned before, estimation of the covariance matrix elements requires an ensemble of samples of the same distribution. Selecting samples using a rectangular window is justified sometimes, if the rectangle size is small enough and loss of resolution is affordable. However, this is never the case for high resolution images of urban areas, e.g. any PS in the estimation window will dominate the covariance or the coherence estimate of the surrounding pixels. Not only will the high resolution
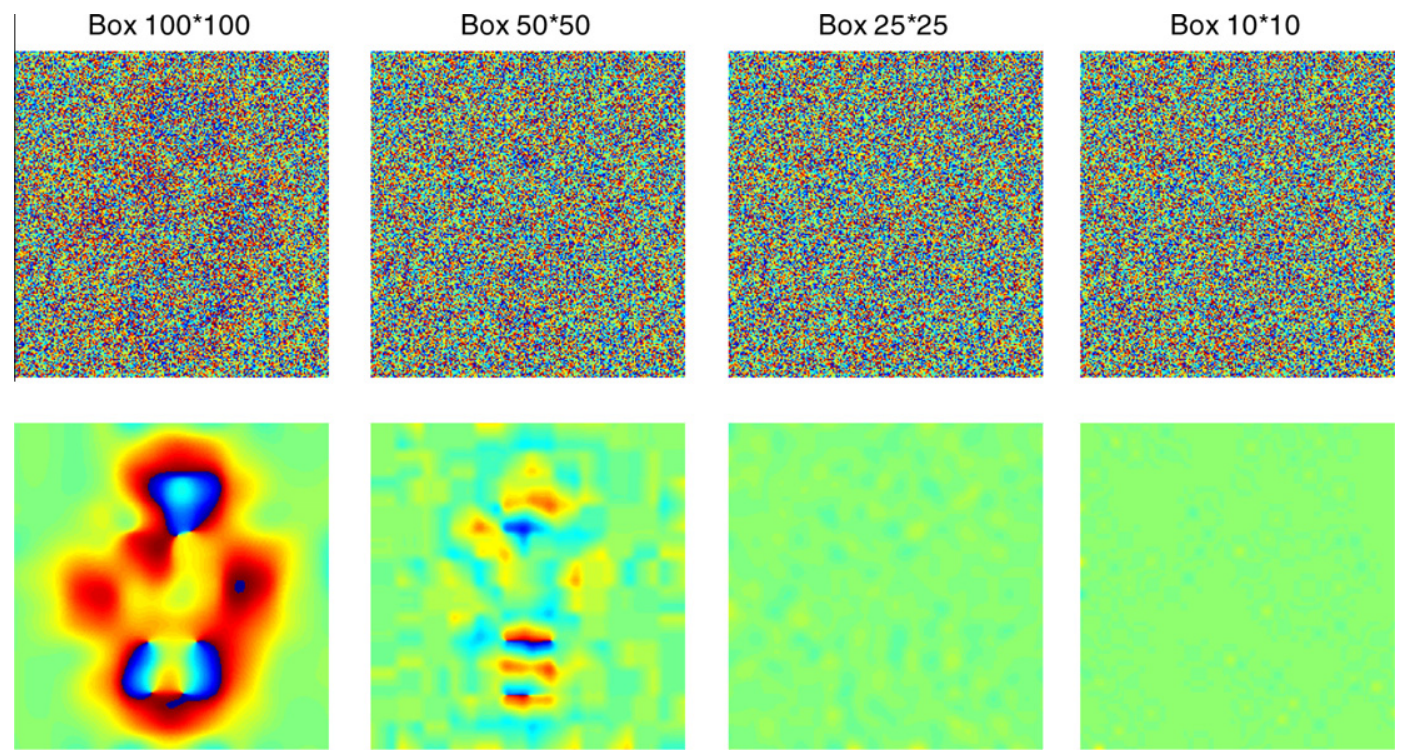

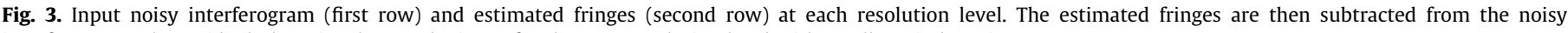
interferogram. The residual phase is taken as the input for the next resolution level with smaller window size. 


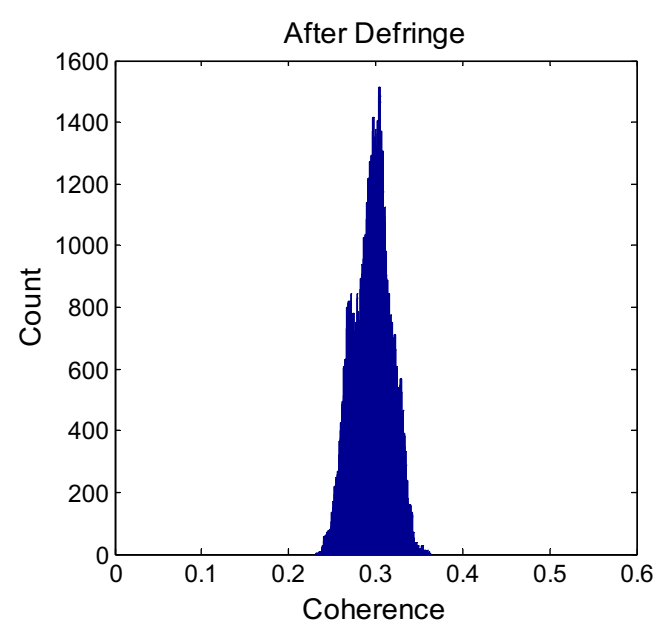

(a)

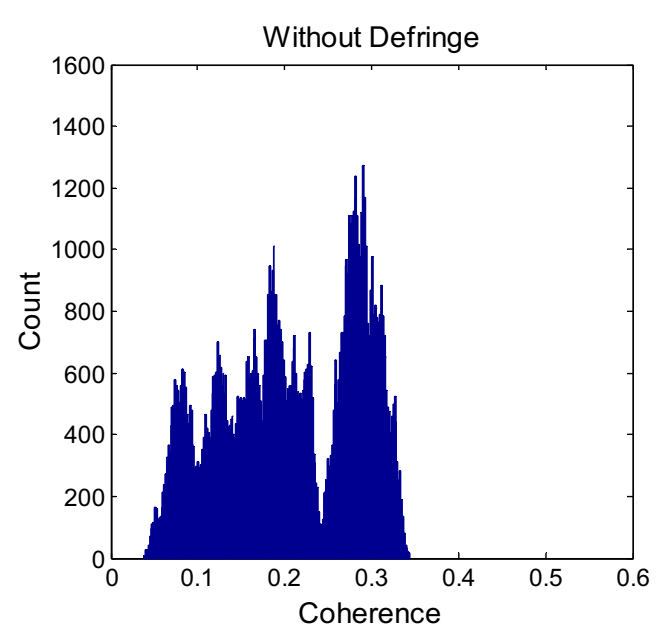

(b)

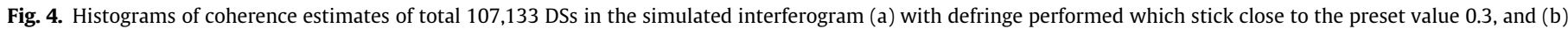
without defringe performed which severely underestimates the coherence.

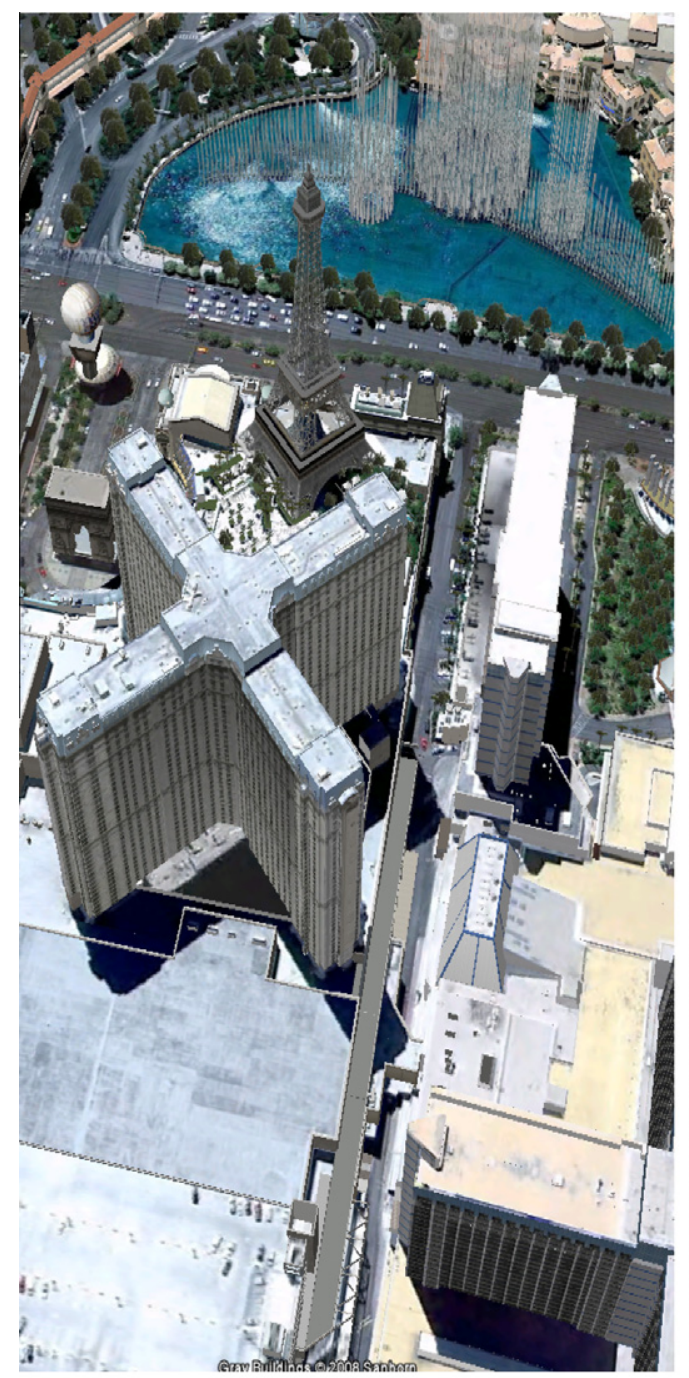

(a)

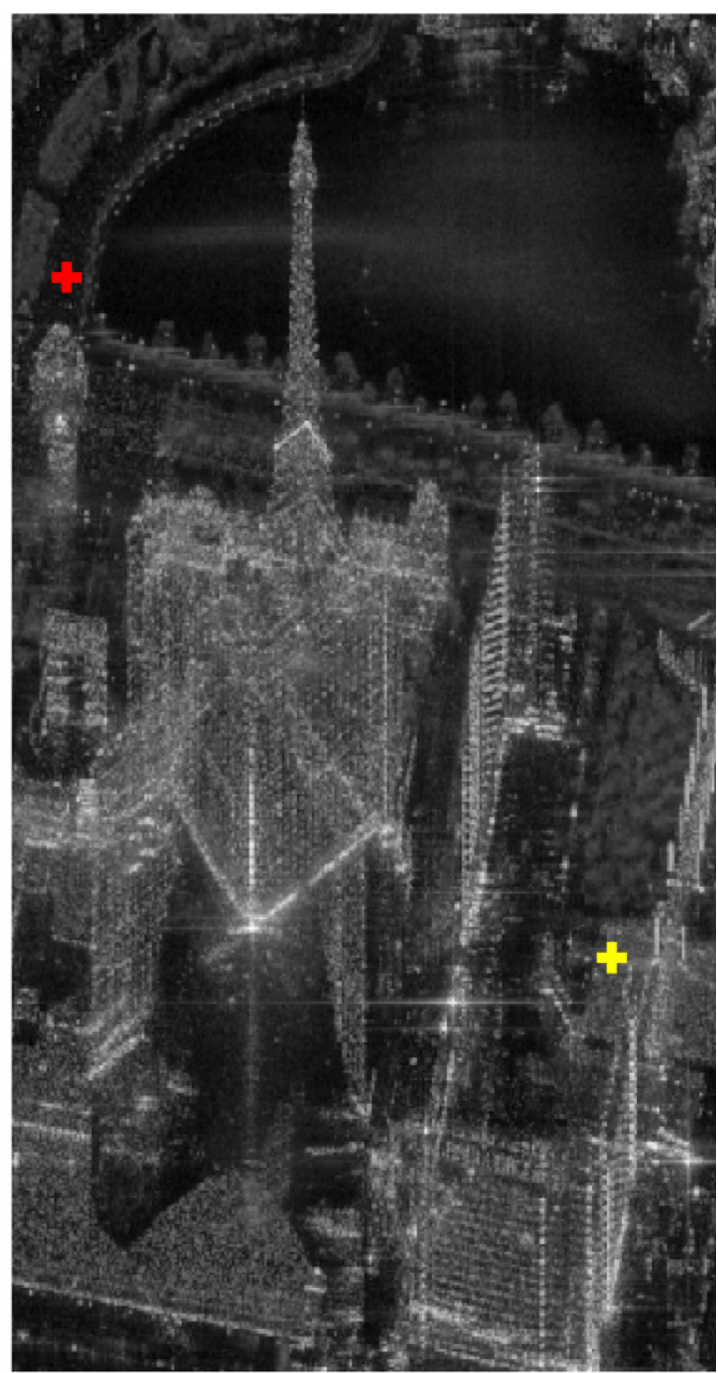

(b)

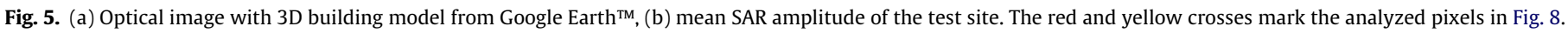



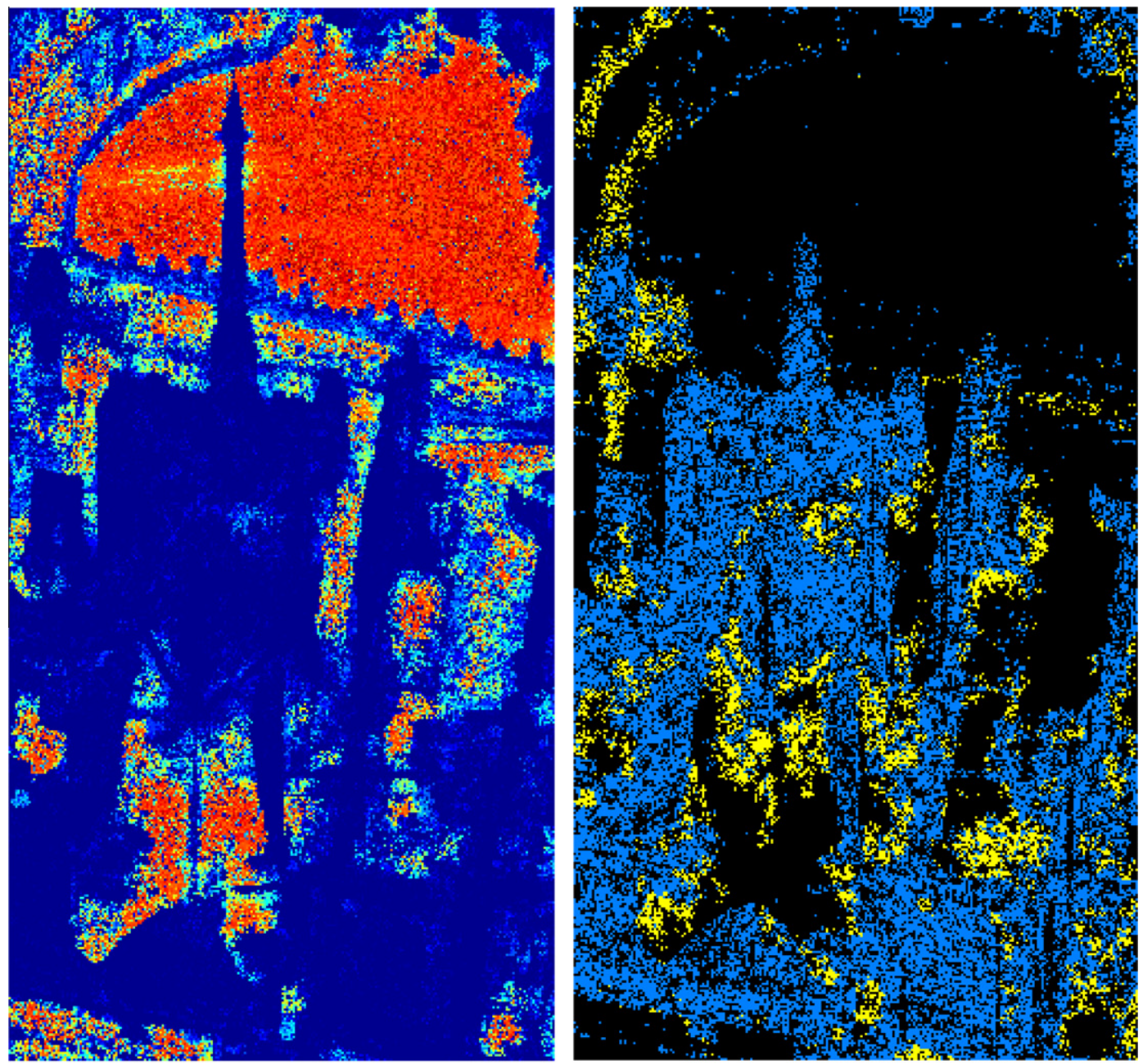

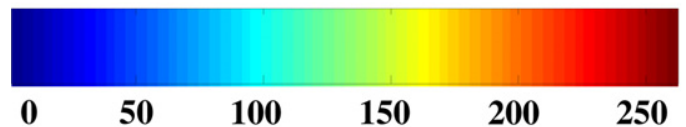

(a)

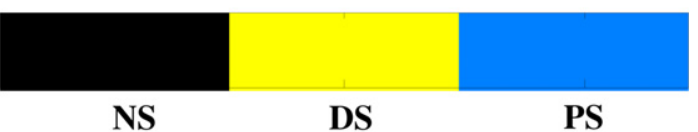

(b)

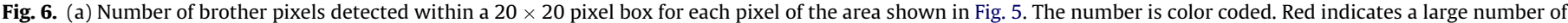
samples and blue indicates a small number, (b) pixel classification map. The blue pixels indicate the PSs, the yellow the DSs, and the black the NS.

be compromised, but the covariance or coherence of a DS close to a PS will also be severely overestimated.

In (Ferretti et al., 2011a) this problem is tackled by introducing the DespecKS method, which detects the samples adaptively by performing the Kolmogorov-Smirnov (KS) test. The KS test compares the maximum distance between the empirical distribution functions (EDFs) of two data sets which, in our case, are the intensities of one pixel throughout the whole stack with another pixel. By setting a threshold on the distance, the hypothesis that the two pixels belong to the same distribution is accepted or rejected. However, the maximum distance of two EDFs usually happens in the midway of the curve, regardless of the shape of the distribution. Therefore, the KS test is not sensitive to unknown distributions with variations on the tails.

We adopt this idea of amplitude based test. Further considering the fact mentioned in the beginning of this section that fully developed speckle can be compromised in high resolution image and Rayleigh distribution is therefore often not satisfied (Jakeman and Pusey, 1976; Jao, 1984), more sophisticated non-parametric tests are more appropriate for our purpose. In (Parizzi and Brcic, 2010), different tests are evaluated. Among them, the AD test offers the best detection rate at a constant false alarm rate. Therefore, we replace the $\mathrm{KS}$ test by the $\mathrm{AD}$ test.

The AD test belongs to the class of quadratic EDF statistics. To compare the probability distributions of pixels $a$ and $b$, we first compute the EDFs $F_{a}(x)$ and $F_{b}(x)$ of the amplitude values of the pixels through the stack. Then the AD test is a weighted L2-norm of the difference between the two EDFs:

$A^{2}=\frac{N}{2} \sum \frac{\left[F_{a}(x)-F_{b}(x)\right]^{2}}{F_{a \cup b}(x)\left[1-F_{a \cup b}(x)\right]}$

where $F_{a \cup b}(x)$ is the EDF of the combined pixel values. $N$ again represents the number of images in our stack. The denominator $F_{a \cup b}(x)\left[1-F_{a \cup b}(x)\right]$ places more weight on the head and tails of the distributions.

The test is performed for every pixel to identify neighboring pixels of the same distribution, i.e. the brother pixels. These form 


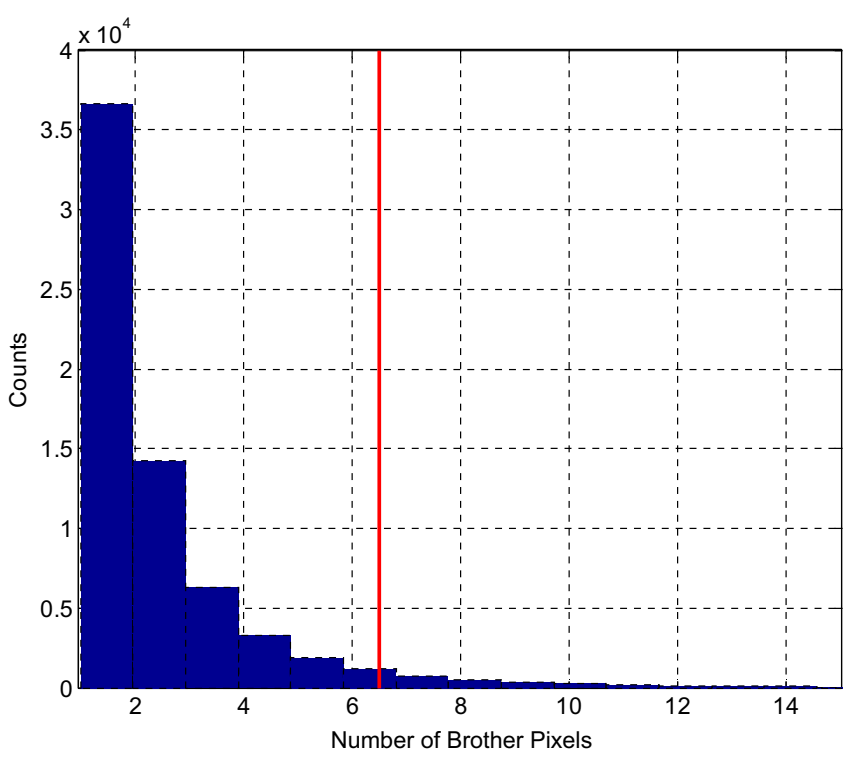

Fig. 7. Histogram of the number of brother pixels of PSs selected by thresholding the amplitude dispersion index. 95\% of the PSs have less than 6 brother pixels. The red line indicates is the cut-off of $95 \%$ of all the PSs.

the estimation window. The window size and shape can vary from one pixel to another. This is unlike segmentation where each pixel belongs to a unique class. In our algorithm one pixel is included in different brotherhoods.

Fig. 1a is an example of adaptive sample selection using the AD test on a stack of high resolution TS-X images of downtown Las Vegas. The test area is near the red cross marked in Fig. $5 \mathrm{~b}$. The red dots in Fig. 1a and $\mathrm{b}$ are our target DS pixel located on the asphalt road between two structures, and the blue ones are the detected brother pixels. As a comparison, samples selected by a rectangular window are marked in Fig. 1b. The adaptive window follows the structure boundary well, while using a rectangle includes all the pixels from both the structures and the road, regardless of their varying amplitudes. The corresponding coherence matrices estimated using the selected samples are shown in the second row of Fig. 1. Since the target pixel is on an asphalt surface having a very low SNR, its coherence is expected to be low. Yet, using the rectangular window, the coherence is heavily overestimated $(>0.7)$, as shown in Fig. 1d. Obviously, we are more confident with the results using the adaptive selection window.

\subsection{Adaptive multi-resolution defringe}

Defringe, also known as phase flattening, is to remove an estimate of the expectation, i.e. a low-pass version, of the interferometric phase before covariance estimation (Eq. (8)). In most of the cases a constant local fringe frequency in range and azimuth directions is assumed. The fringe frequency is found by simply searching the maximum coefficient in the Fourier transform of local patches (Bamler and Hartl, 1998; Zebker and Chen, 2005).

Yet the assumption of a constant frequency is not always valid, especially in urban areas with fast varying topography. And the local fringe frequency cannot be correctly estimated at areas where decorrelation noise dominants. We make use an adaptive multi-resolution defringe algorithm, firstly introduced for phase unwrapping (Davidson and Bamler, 1999). It overcomes the aforementioned two problems by on one hand demodulating the interferogram multiple times by estimating local fringes in patches of difference sizes, and on the other hand taking into account only the pixels with sufficiently high coherence.
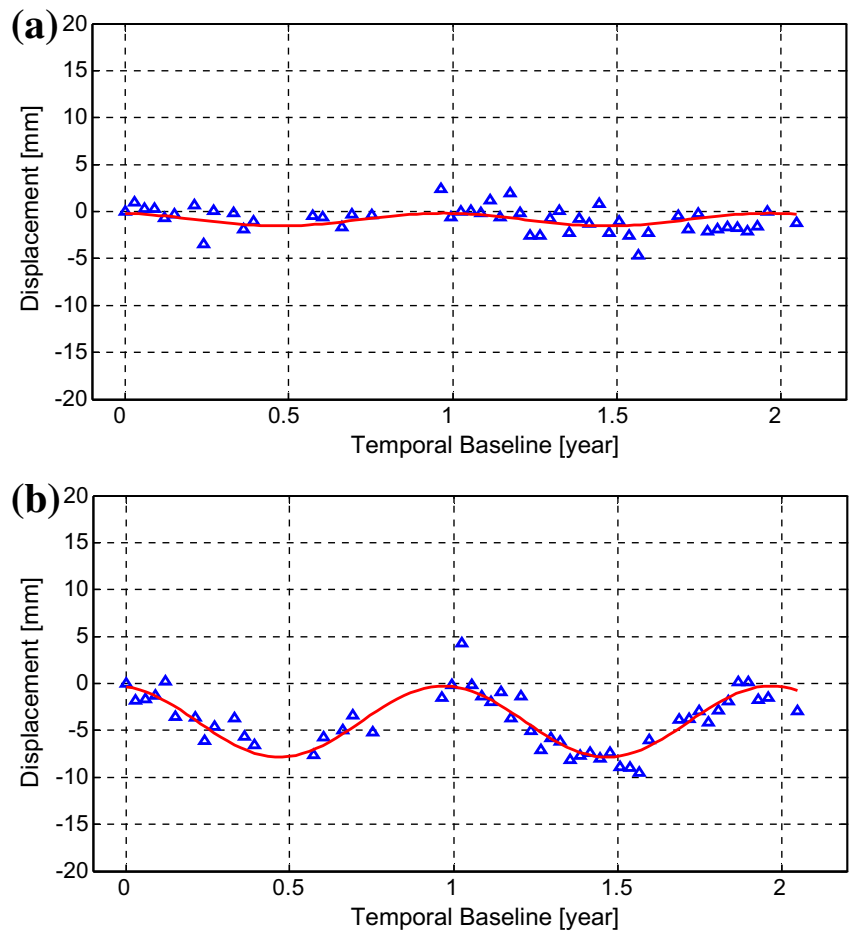

Fig. 8. (a) Phase history of one DS located on the ground, indicated by the red cross in Fig. 5 (b). Its coherence equals 0.16. This pixel experiences no significant deformation, (b) phase history of another DS located on the roof of a building, which undergoes a seasonal motion with $4.5 \mathrm{~mm}$ in amplitude. Its coherence is 0.29 . This pixel is indicated as the yellow cross in Fig. 5 (b).

We test this algorithm on a simulated interferogram of $600 \times 600$ pixels. The simulated interferometric phase is shown in Fig. 2b. The pixels in the interferogram are either DS with coherence equal to 0.3 , i.e. SNR $=-3.7 \mathrm{~dB}$ in both SLC images, or noise (NS) with coherence close to 0 . We uniformly distribute $30 \%$ of the pixels as DS, and the rest $70 \%$ to be NS. This setting simulates the worst real case scenario where no high coherence scatterer is available, but only low coherence targets are sparsely scattered in the scene. The noise-free and noisy interferogram are collocated in Fig. 2a and b. The fringe pattern is hardly distinguishable in the noisy interferogram.

We apply the multi-resolution algorithm to the noisy interferogram. Fig. 3 shows the input and estimated fringes at each resolution level. Starting from the largest patch size, the fringe frequency is estimated using only the DSs in a patch. The interferogram is then demodulated, and passed to the next resolution level. In our test, we set four resolution levels: $100 \times 100,50 \times 50,25 \times 25$, and $10 \times 10$ pixels. The summation of the phase contributions at all the resolution levels gives the final result in Fig. 2c. For comparison, Fig. 2d shows the result for the usual fixed window size and without employing the pre-selection of coherent pixels. This method completely fails to reconstruct the original interferometric phase, while the multi-resolution algorithm reduces the estimation error tremendously and retains higher correlation with the noise-free interferogram.

After defringing, the coherences of all the DSs in the interferogram are evaluated. The results show consistent and accurate solutions with respect to the preset coherence of 0.3 . 99\% of the results lie in the interval of $0.3 \pm 0.05$. This result is compared to the coherence estimated without defringe. Fig. 4 plots the histogram of the estimated coherence, with defringe and without defringe, of total 107,133 DSs. It is obvious that the coherence is severely underestimated if defringe is not performed. 


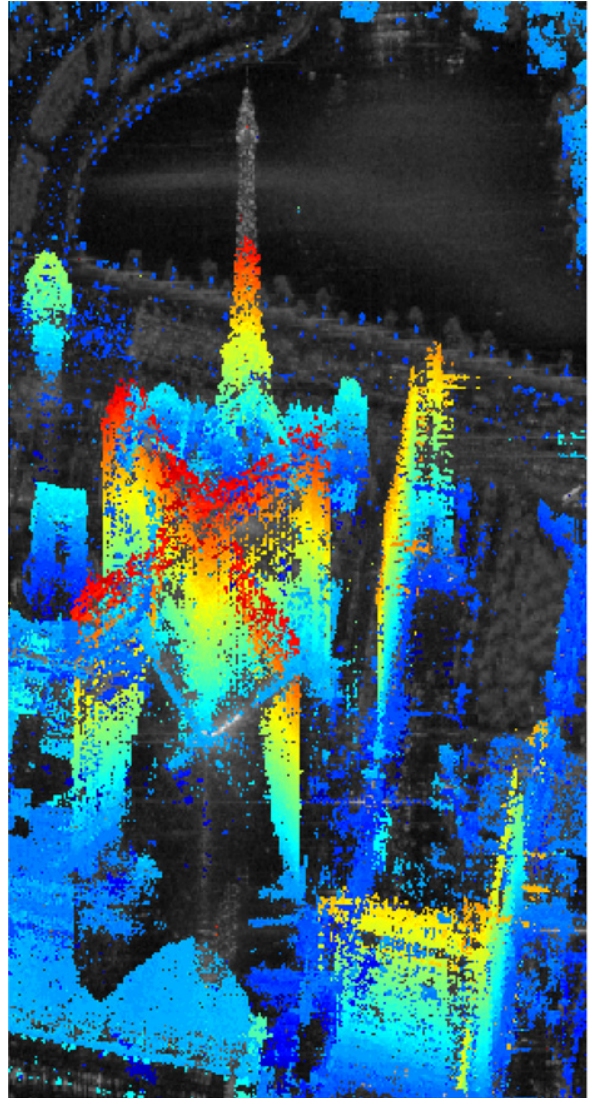

(a)

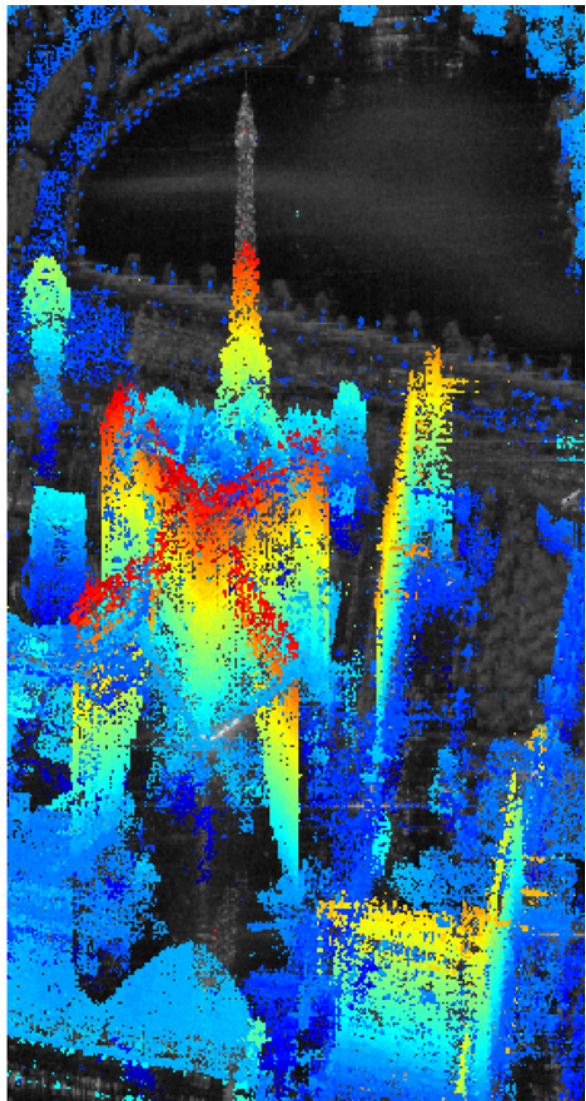

(b)

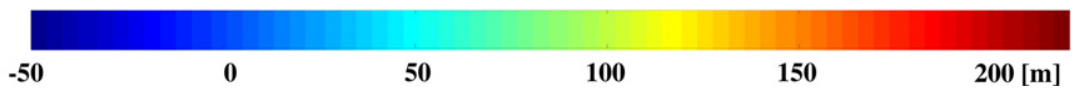

Fig. 9. Estimated elevation at (a) only PSs, and (b) both PSs and DSs.

\section{Parameters estimation}

\subsection{Maximum likelihood estimator}

We use MLE for parameter estimation for each DS pixel. We make use of the objective function developed in (De Zan and Rocca, 2005):

$\{\hat{h}, \hat{a}\}=\arg \max \left\{\frac{\exp \left(-\mathbf{i}^{\mathrm{H}} \mathbf{\Phi}(h, a) \mathbf{C}_{0}^{-1} \mathbf{\Phi}(h, a)^{\mathrm{H}} \mathbf{i}\right)}{\operatorname{det}\left|\mathbf{C}_{0}\right| \pi^{N}}\right\}$

where $i$ is the complex measurements vector; $\boldsymbol{\Phi}$ is diagonal matrix containing the modeled phase; $\mathbf{c}_{0}$ is absolute value of the covariance matrix; $h, a$ is height above reference surface, line-of-sight (LOS) motion parameter such as linear deformation velocity and seasonal displacement amplitude and $N$ is the number of images

In the likelihood function, the covariance matrix $\mathbf{C}$ is decomposed to $\boldsymbol{\Phi}(h, a) \mathbf{C}_{0}^{-1} \mathbf{\Phi}(h, a)^{\mathrm{H}}$. The diagonal matrix $\boldsymbol{\Phi}$ contains the phase of the model. The denominator $\operatorname{det}\left|\mathbf{C}_{0}\right| \pi^{N}$ can be ignored: it is a constant for each pixel.

\section{Experiments}

\subsection{Test site 1 - Paris Las Vegas hotel}

We apply the proposed algorithm to a test site about $500 \times 500 \mathrm{~m}$ centered at Paris Las Vegas hotel in downtown Las Vegas cropped from a stack of co-registered 50 TS-X high resolution spotlight images. Fig. 5a and b are the optical image from Google
Earth $^{\mathrm{TM}}$ and the mean amplitude image of the test area, respectively. In this test case, the area consists of different types of scattering objects, i.e. partly building façades (probable PS candidates), partly asphalt roads and rough surface (probable DS candidates), and a large fraction of totally decorrelated scatterers (e.g. the fountain on the top of the scene, and vegetated areas).

The pre-processing, including amplitude calibration and atmospheric phase screen (APS) correction, is performed using the PSI-GENESIS system from the German Aerospace Center (DLR) (Kampes, 2006; Adam et al., 2003). We firstly classify all the pixels to PS, DS, or NS, by jointly considering the coherence and the number of brother pixels. Based on the classification, we process PSs using the standard PSI technique, and DSs using the proposed algorithm.

\subsection{Pixel classification}

To classify PS, DS and NS, we firstly identify the PSs by making use of the property explained in (Ferretti et al., 2011a) that most of the PSs possess very few brother pixels. Showing in Fig. 6, the number of the brother pixel map, most of the building structures show up in blue which indicates a small number of brother pixels, in contrast to the orange color for the rest of the image. Statistics tell that $95 \%$ of the PSs (selected by thresholding on amplitude dispersion index) have less than 6 samples (see Fig. 7). Secondly, the DSs are distinguished from the rest of the pixels by thresholding on the coherence. The threshold is set at the unbiased lower limit of the coherence estimator in Eq. (4) using 200 looks. The final classifica- 


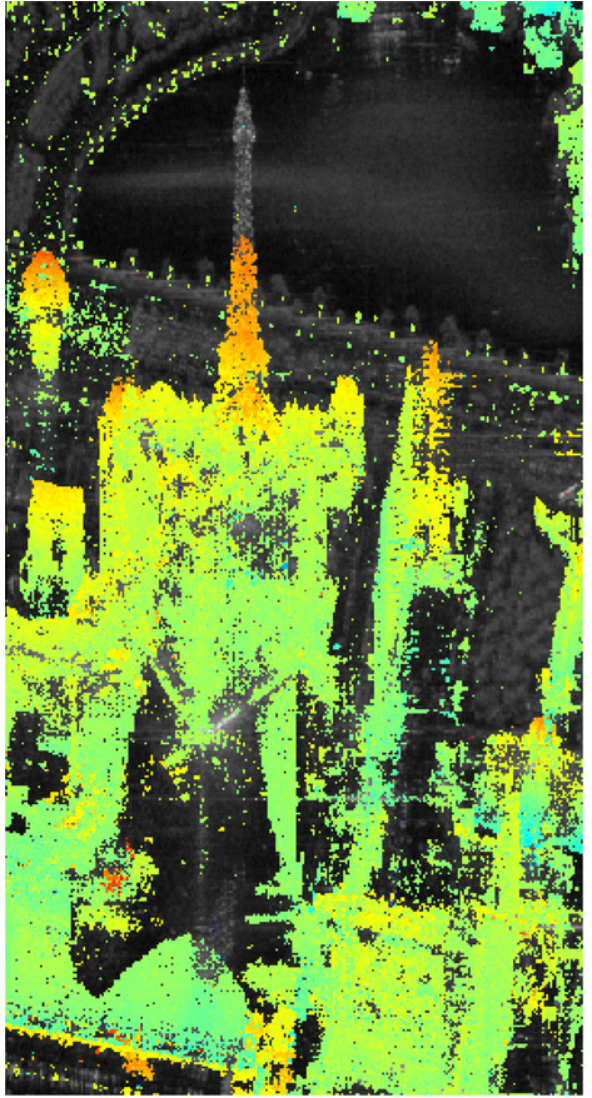

(a)

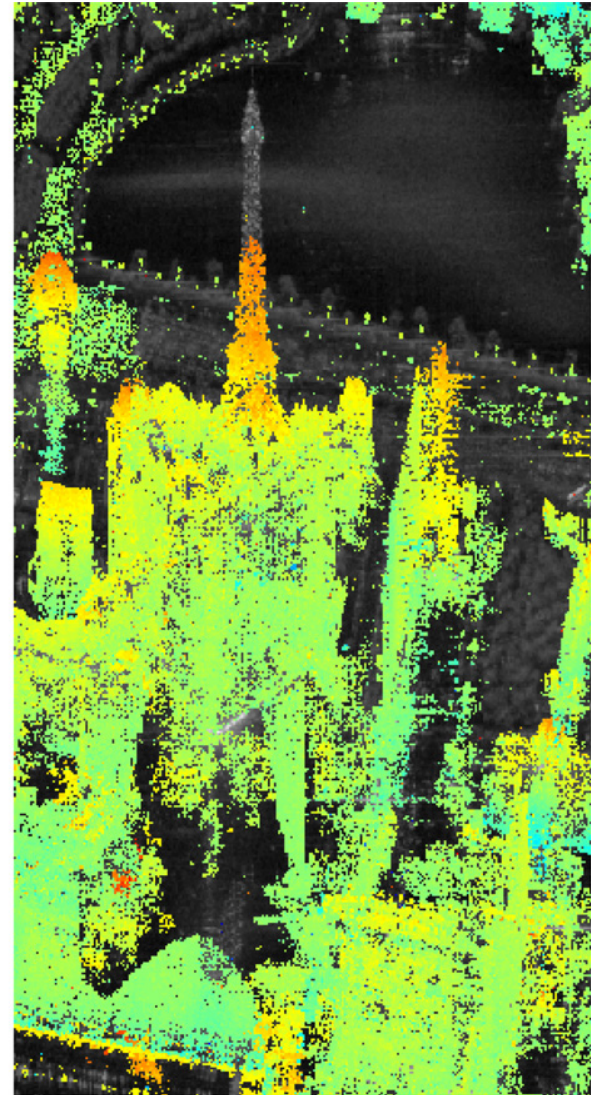

(b)

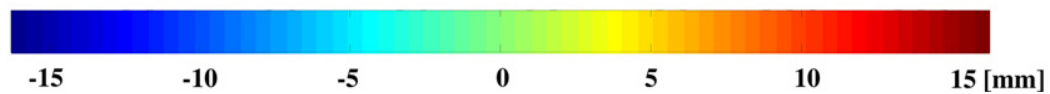

Fig. 10. Estimated seasonal deformation amplitude at (a) only PSs, and (b) both PSs and DSs.

tion is shown in Fig. 6b, where PS, DS, and NS are marked as different colors. In this test site, the number of DSs is about $20 \%$ of PSs.

\subsection{Retrieved phase history}

According to the classification map, we estimate the covariance matrix for each DS after sorting all the interferograms along the temporal baseline. The phases of the covariance matrix's first column represent the expected interferometric phases referring to the first image. Since defringe was applied prior to the covariance estimation, the pre-removed phase was added back to get the final phase history of each pixel. Fig. 8 plots the phase histories of two DSs (indicated as red and yellow crosses in Fig. 5b). Seen from that, the first one is located on the asphalt road at ground level, with presumably no significant deformation. The reflectivity of such material is generally very low, as we can see the surrounding pixels are consistently dark. For this pixel, the coherence is 0.16 , equivalent to an average SNR of $-7.1 \mathrm{~dB}$. Its phase history shows almost no deformation throughout the two years' time span. The second one is on the roof of a building, possibly subject to thermal expansion. It has a better coherence of 0.29 , corresponding to an average SNR of $-4 \mathrm{~dB}$. It undergoes a seasonal deformation of $4.5 \mathrm{~mm}$ in amplitude. The red curves in the figures are the fitting of the sinusoidal deformation model.

\subsection{Estimated parameters}

Following the retrieved phase history, we estimate the elevation and seasonal motion amplitude for each DS. The result is combined with the estimates from PSs using the standard PSI procedures. All the estimates are referred to a single reference point. Figs. 9a and 10a give the estimates from PSs only, and Figs. $9 \mathrm{~b}$ and $10 \mathrm{~b}$ are PSs and DSs combined. We see a linear gradient from the base of the buildings to the top in Fig. 9, with blue indicating low elevation, and red for high. A similar gradient can also be observed in the seasonal motion amplitude estimate in Fig. 10. The seasonal motion amplitude becomes more positive as the elevation gets higher representing displacement towards the sensor. This is mostly caused by the natural expansion and contraction of the building itself, but also subject to the effect of height dependent APS to a maximum of about $1.5 \mathrm{~mm}$ per $100 \mathrm{~m}$ in vertical height (Cong and Eineder, 2012).

The PSs already provide very good coverage over most parts of the building façades, except for the upper half of the tower due to its very complex motion. The DSs give extra information over the rest of the image. The contributions of DSs are scattered over the whole area, including areas on the upper left corner and area close to the center of the image. Most of the DSs are on the ground level, indicated by the blue color. Their deformation amplitudes are generally small (green). It is worthy to point out that seemingly reliable estimates also found in the shadow area. They are "ghost scatterers" caused by mirror reflections of the façade at the ground (Auer et al., 2011). The variances of these DS estimates are comparable with those of PS estimates, although the coherences of these DSs are much lower. Nevertheless, the MLE of the phase history of DS is obtained by covariance matrix estimation based on many samples. The residual of the phase history to the sinusoidal model is about $1-2 \mathrm{~mm}$, thus providing a reliable estimation of the model parameters. 


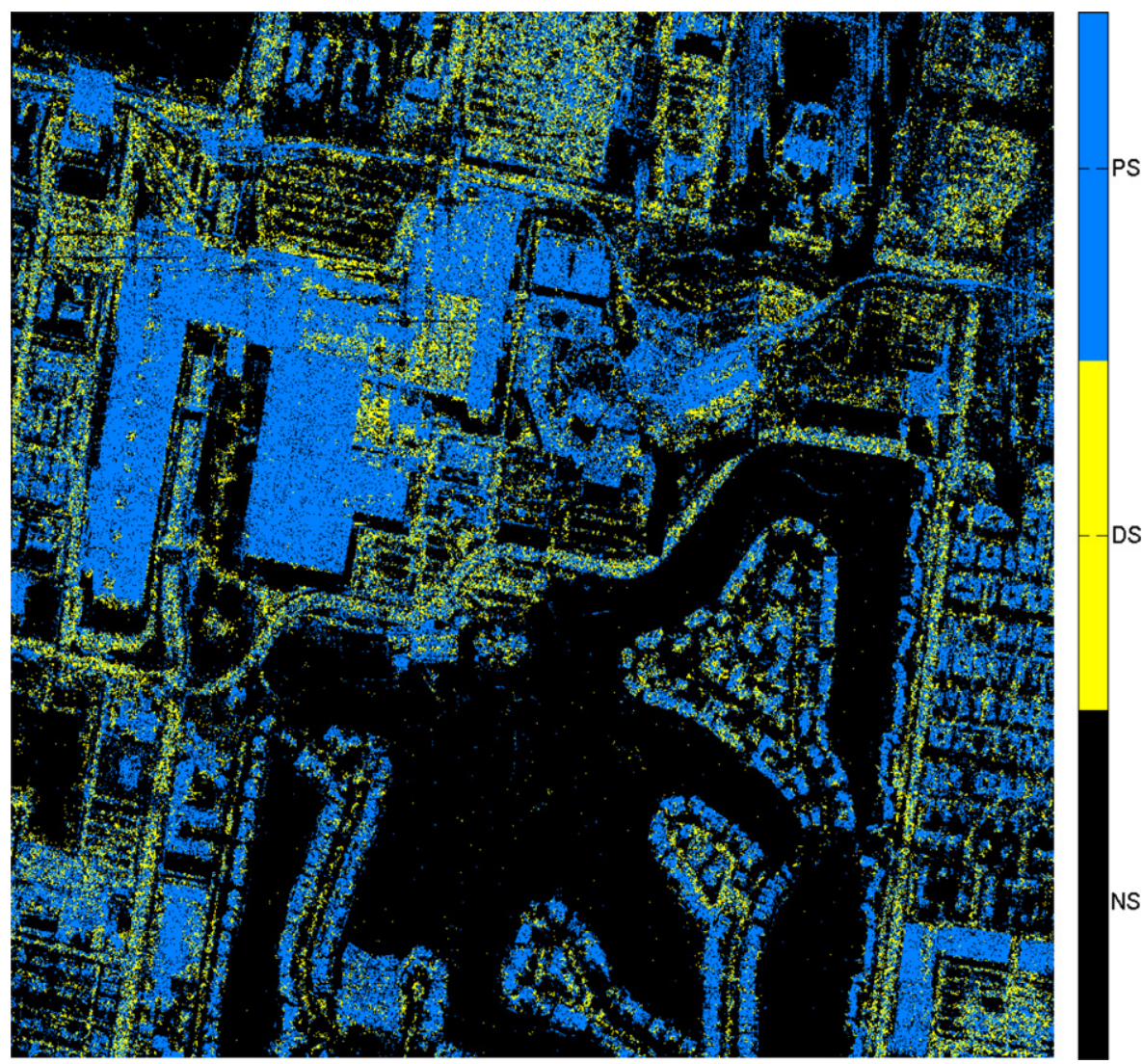

Fig. 11. Pixel classification map. The blue pixels indicate the PSs, the yellow the DSs, and the black the NS.

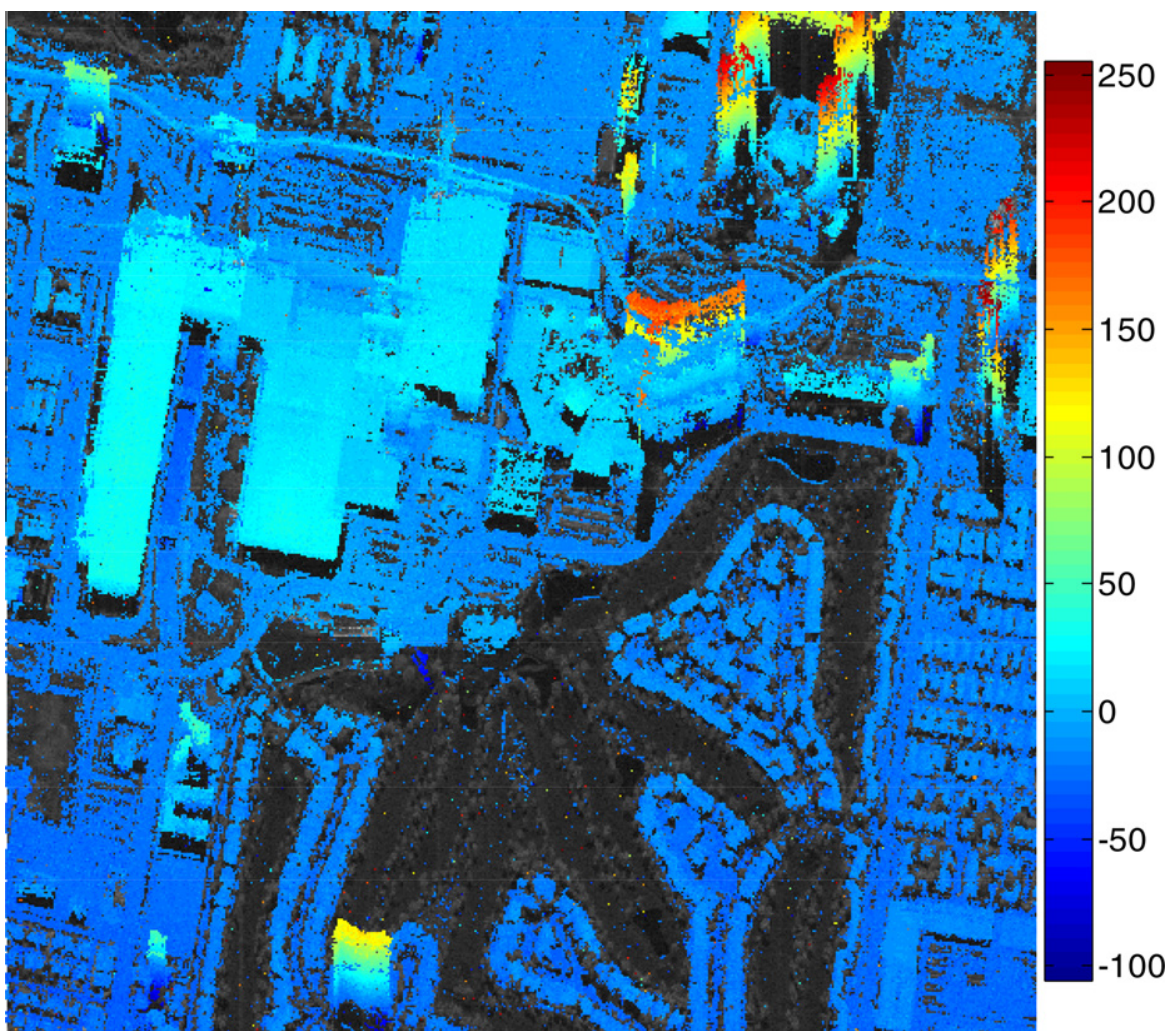

Fig. 12. Estimated elevation of both PSs and DSs of the second test site. The unit is in meter. 


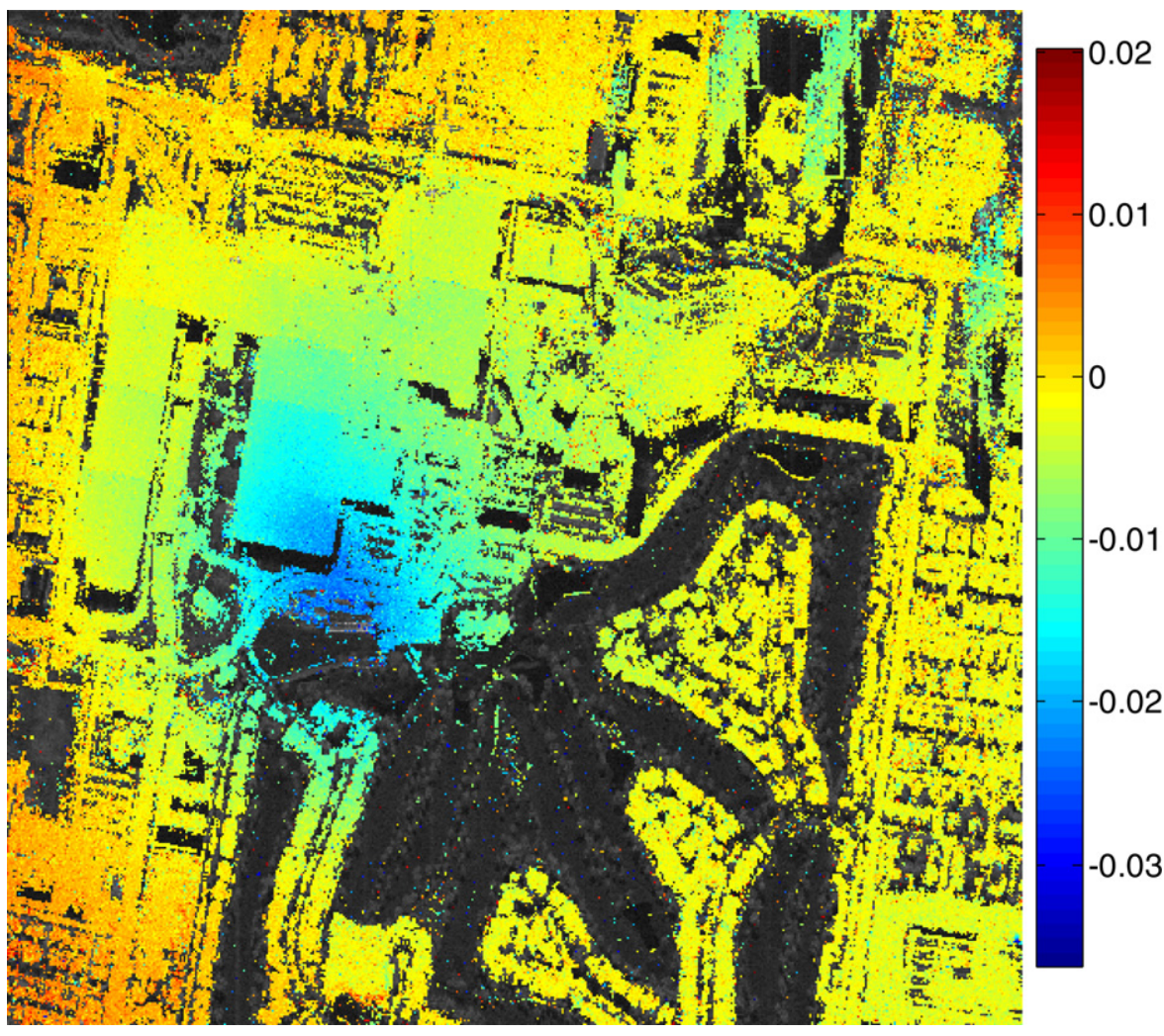

Fig. 13. Estimated linear deformation rate of both PSs and DSs. The unit is in $\mathrm{m} / \mathrm{year}$.

\subsection{Test site 2 - Las Vegas convention center}

A larger area about $1 \times 2 \mathrm{~km}$ around the Las Vegas convention center is chosen as the second test site due to a known subsidence discovered in (Zhu and Bamler, 2011). The same processing procedures are applied. The pixel classification is shown in Fig. 11. Blue color (PS) fills up most of the building structures, while yellow (DS) appears mostly on the road. The park located on the lower half of the image is almost black due to temporal decorrelation of vegetation. In Figs. 12 and 13, the estimation results of PSs and DSs combined are demonstrated. The elevation estimates look consistent and reliable for both PS and DS, except part of the building façades due to the layover problem. The linear deformation rate estimates show a subsidence pattern centered at Las Vegas convention center with a maximum of about $15 \mathrm{~mm} /$ year.

\section{Conclusion and outlook}

This article presents optimal DS phase history and model parameters retrieval in urban areas using very high resolution TS-X spotlight data. To preserve the high spatial resolution, particular focus is put on the phase history retrieval for each DS pixel. We emphasize the critical problem of accurate covariance matrix estimation. A solution is provided by using adaptive sample selection and a multi-resolution defringe method. The adaptive sample selection method is proven to be an essential procedure in the covariance matrix estimation. The AD test performs well in distinguishing targets of different scattering characteristics. The adaptive multi-resolution defringe algorithm outperforms the method with a fixed window size in terms of fringe estimation accuracy and robustness to noise. The effectiveness of the proposed method is tested using simulated and real TS-X data. Periodic seasonal motion histories of sample DS have been derived. The residuals to the sinusoidal model were in the order of $1-2 \mathrm{~mm}$.
In the future, as a more general solution, we will improve the DS covariance matrix estimation using a "fuzzy brotherhood" concept, i.e. using surrounding pixels with weightings derived from a statistical test instead of using a fixed threshold. The improved algorithm will be applied to non-urban area, and possible mixed single- and repeat-pass data stacks.

\section{Acknowledgements}

This work is supported by International Graduate School of Science and Engineering (IGSSE), Technische Universität München (IGSSE Project 6.08: "4D City"), and the German Research Foundation (DFG, Förderkennzeichen BA2033/3-1). The authors would like to thank Alessandro Parizzi from DLR for valuable discussions and one of the reviewers who pointed out the possible misinterpretation of height dependent APS as seasonal motion.

\section{References}

Adam, N., Kampes, B., Eineder, M., Worawattanamateekul, J., Kircher, M., 2003. The development of a scientific permanent scatterer system. In: Proc. ISPRS Hannover Workshop, Hannover, Germany, 6-8 October, pp 1-6 (on CD-ROM).

Auer, S., Gernhardt, S., Bamler, R., 2011. Ghost persistent scatterers related to multiple signal reflections. IEEE Geoscience and Remote Sensing Letters 8 (5), 919-923.

Bamler, R., Hartl, P., 1998. Synthetic aperture radar interferometry. Inverse Problems 14 (4), R1-R54.

Casu, F., Manzo, M., Berardino, P., Fornaro, G., 2005. The SBAS approach for earth surface deformation analysis. Atti della Fondazione Ronchi, Anno LX, no. 4, pp. 611-618.

Cong, X., Eineder, M., 2012. Volcano deformation measurement using persistent scatterer interferometry with atmospheric delay corrections. In: Proc. EUSAR Conference 2012, Nuremberg, Germany, 23-26 April (on CD-ROM).

Davidson, G.W., Bamler, R., 1999. Multiresolution phase unwrapping for SAR interferometry. IEEE Transactions on Geoscience and Remote Sensing 37 (1) 163-174.

De Zan, F., Rocca, F., 2005. Coherent processing of long series of SAR images. In: Proc. IGARSS 2005, Seoul, Korea, 25-29 July, pp. 1987-1990. 
Ferretti, A., Fumagalli, A., Novali, F., Prati, C., Rocca, F., Rucci, A., 2011a. A new algorithm for processing interferometric data-stacks: SqueeSAR. IEEE Transactions on Geoscience and Remote Sensing 49 (9), 3460-3470.

Ferretti, A., Fumagalli, A., Novali, F., Prati, C., Rocca, F., Rucci, A., 2011b. Exploitation of temporary coherent scatterers in SqueeSAR analyses. In: Proc. IGARSS 2011, Vancouver, Canada, 24-29 July (on CD-ROM).

Ferretti, A., Prati, C., Rocca, F., 2001. Permanent scatterers in SAR interferometry. IEEE Transactions on Geoscience and Remote Sensing 39 (1), 8-20.

Ferretti, A., Bianchi, M., Prati, C., Rocca, F., 2005. Higher-order permanent scatterers analysis. EURASIP Journal on Applied Signal Processing 2005 (20), 32313242 .

Fornaro, G., Reale, D., Serafino, F., 2009. Four-dimensional SAR imaging for height estimation and monitoring of single and double scatterers. IEEE Transactions on Geoscience and Remote Sensing 47 (1), 224-237.

Jakeman, E., Pusey, P.N., 1976. A model for non-Rayleigh sea echo. IEEE Transactions on Antennas and Propagation 24 (6), 806-814.

Jao, J.K., 1984. Amplitude distribution of composite terrain radar clutter and the Kdistribution. IEEE Transactions on Antennas and Propagation 32 (10), 10491062.

Kampes, B., 2006. Radar Interferometry: Persistent Scatterers Technique, first ed. Springer Netherlands, Dordrecht.
Lombardini, F., 2005. Differential tomography: a new framework for SAR interferometry. IEEE Transactions on Geoscience and Remote Sensing 43 (1), 37-44.

Parizzi, A., Brcic, R., 2010. Adaptive InSAR stacks multi-looking exploiting amplitudes statistics - a comparison between different techniques and practical results. IEEE Geoscience and Remote Sensing Letters 8 (3), 441-445.

Reed, I.S., Mallett, J.D., Brennan, L.E., 1974. Rapid convergence rate in adaptive arrays. IEEE Transactions on Aerospace and Electronic Systems 10 (6), 853-863.

Seymour, M., Cumming, I., 1994. Maximum likelihood estimation for SAR interferometry. In: Proc. IGARSS 1994, Pasadena, USA, 8-12 August, pp. 22722275 .

Touzi, R., Lopes, A., Bruniquel, J., Vachon, P.W., 1999. Coherence estimation for SAR imagery. IEEE Transactions on Geoscience and Remote Sensing 37 (1), 135-149.

Zebker, H.A., Chen, K., 2005. Accurate estimation of correlation in InSAR observations. IEEE Geoscience and Remote Sensing Letters 2 (2), 124-127.

Zhu, X., Bamler, R., 2010. Very high resolution spaceborne SAR tomography in urban environment. IEEE Transactions on Geoscience and Remote Sensing 48 (12), 4296-4308.

Zhu, X., Bamler, R., 2011. Let's do the time warp: multicomponent nonlinear motion estimation in differential SAR tomography. IEEE Geoscience and Remote Sensing Letters 8 (4), 735-739. 